\title{
The contribution of hydrodynamic processes to calcite dissolution rate spectra: measurements and simulations
}

\author{
MARIETTE WOLTHERS ${ }^{1}$, PRIYANKA AGRAWAL ${ }^{2}$, TILL \\ BOLLERMANN $^{3}$, OLEG ILIEV ${ }^{4}$, CORNELIUS FISCHER $^{5}$ \\ AND AMIR RAOOF ${ }^{6}$ \\ ${ }^{1}$ Earth Sciences, Utrecht University \\ ${ }^{2}$ Utrecht University \\ ${ }^{3}$ Institute of Resource Ecology, Reactive Transport Department \\ ${ }^{4}$ Fraunhofer Institute for Industrial Mathematics ITWM \\ ${ }^{5}$ Helmholtz-Zentrum Dresden-Rossendorf (HZDR), Institute of \\ Resource Ecology, Reactive Transport Department \\ ${ }^{6}$ Department of Earth Sciences, Utrecht University \\ Presenting Author: m.wolthers@uu.nl
}

Recent studies on the dissolution rate of microscopically rough calcite surfaces have shown lateral variations in dissolution rate. The resulting dissolution rate spectra are considered to reflect an intrinsic variability of crystal surface reactivity, distinct from extrinsic factors, such as inhibitors or $\mathrm{pH}$ variations (Fischer et al., 2014). We have investigated if hydrodynamic effects at microscopically rough calcite surfaces yield lateral variations in dissolution rate comparable to observed rate spectra. We simulated the dissolution of a calcite surface at the micrometer scale, using measured surface topographies, and compared the resulting rate spectra to the measured rate spectra using Vertical Scanning Interferometry at the same reactive-flow conditions.

Simulating dissolution of the microscopically rough calcite did not produce similarly wide dissolution rate spectra like those observed experimentally when only flow heterogeneity near the real crystal topography was combined with a single dissolution rate (Fig. 1). Simulated and measured rate spectra agreed only when rate spectra were imposed explicitly into the simulations. This study therefore implies that, for the reactive-flow regimes under consideration, the heterogeneity and topography of the crystal surface and the resulting heterogeneity in the free energy landscape at the surface play a major role in controlling dissolution rate spectra.

Reference: Fischer, Kurganskaya, Schäfer and Luittge (2014), Appl. Geochem. 43, 132-157.

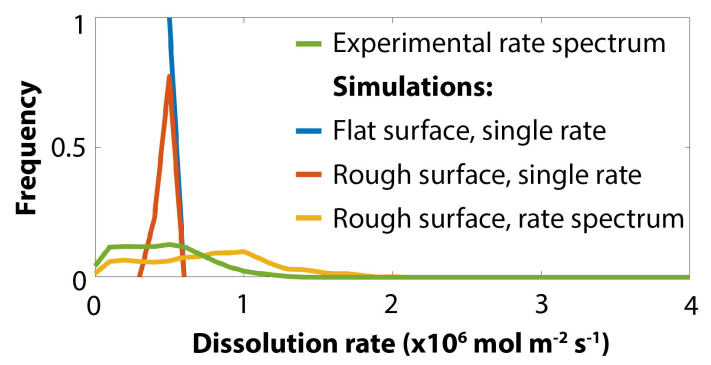

